\title{
Axl is a novel target of celastrol that inhibits cell proliferation and migration, and increases the cytotoxicity of gefitinib in EGFR mutant non-small cell lung cancer cells
}

\author{
YOUN JU LEE ${ }^{1 *}$, SO-YOUNG KIM ${ }^{2 *}$ and CHUHEE LEE ${ }^{3}$ \\ ${ }^{1}$ Department of Pharmacology, College of Medicine, Catholic University of Daegu, Daegu 42472; \\ ${ }^{2}$ Department of Pharmacology, School of Medicine, Dongguk University, Gyeongju, Gyeongbuk 38066; \\ ${ }^{3}$ Department of Biochemistry and Molecular Biology, School of Medicine, Yeungnam University, \\ Daegu 42415, Republic of Korea
}

Received August 24, 2018; Accepted February 7, 2019

DOI: $10.3892 / \mathrm{mmr} .2019 .9957$

\begin{abstract}
Gefitinib, an epidermal growth factor receptor tyrosine kinase inhibitor (EGFR-TKI) is an excellent therapeutic agent to treat EGFR mutation-positive non-small cell lung cancer (NSCLC). However, the initial response decreases as chemoresistance develops. In the present study, gefitinib-resistant EGFR mutant NSCLC PC-9/GR cells were established to examine the characteristics and mechanisms associated with chemoresistance. Axl expression in PC-9/GR cells was transcriptionally upregulated, since Axl protein and mRNA expression levels were identified to be increased according to western blot analysis and reverse transcription polymerase chain reaction results. The inhibitory effect of celastrol on Axl protein expression level, cell viability and clonogenicity were identified in parental and gefitinib-resistant PC-9 cells. In addition, treatment of PC-9/GR cells with celastrol and gefitinib in combination was demonstrated to synergistically suppress Axl protein expression level, cell proliferation and migration. Taken together, upregulation of Axl expression seems to be associated with chemoresistance of PC-9/GR cells. Furthermore, celastrol targets Axl to exert its anticancer effects in order to increase the susceptibility of PC-9/GR cells to gefitinib and overcome chemoresistance.
\end{abstract}

\section{Introduction}

Lung cancer remains the chief cause of cancer death worldwide (1). Most lung cancer is non-small cell lung cancer

Correspondence to: Dr Chuhee Lee, Department of Biochemistry and Molecular Biology, School of Medicine, Yeungnam University, 170 Hyun-Chung Ro, Daegu 42415, Republic of Korea

E-mail: chlee2@ynu.ac.kr

${ }^{*}$ Contributed equally

Key words: Axl, celastrol, chemoresistance, epidermal growth factor receptor, gefitinib, non-small-cell lung carcinoma
(NSCLC) corresponding to more than $85 \%$ of lung cancers (2). Platinum-based chemotherapy has been prescribed as a first-line treatment for NSCLC patients but yielded limited outcomes in terms of overall survival and 5-year survival rate (3). In the past decade, there has been dramatic paradigm shift in the treatment of NSCLC, since constitutive activation of epidermal growth factor receptor (EGFR) have been discovered and verified to be closely correlated with the initiation, progression and poor prognosis of NSCLC (4-6).

EGFR is a member of the HER/ErbB family of receptor tyrosine kinase (RTK) composed of four closely related RTKs: EGFR (ErbB1), HER2/neu (ErbB2), HER3 (ErbB3) and HER4 (ErbB4) $(7,8)$. These receptors are located in the plasma membrane and have similar structural features, that are extracellular ligand-binding domain, a single transmembrane region, and a cytoplasmic tyrosine kinase domain (9). A number of ligands including epidermal growth factor (EGF) and transforming growth factor- $\alpha$, bind to EGFR, which induce receptor dimerization and autophosphorylation and/or transphosphorylation of tyrosine residues in the cytoplasmic domain (10). Phosphorylation of EGFR evokes its kinase activity, which subsequently activates several downstream signaling molecules, including phospholipase C- $\gamma$, Ras, phosphatidylinositol-3 kinase (PI-3K), Janus kinase (JAK), signal transducers and activators of transcription (STAT)s, protein kinase B (AKT/PKB) and mitogen-activated protein kinases (MAPK) involved in regulation of many cellular processes, including cell proliferation, survival, migration and apoptosis (11). The oncogenic mechanisms to drive EGFR constitutively active are gene amplification and/or gene mutations. Two most common EGFR-activating mutations are short in-frame deletions in exon 19, E746-A750, and point mutation in exon 21, L858R, which have been demonstrated to be useful biomarkers and therapeutic targets to treat NSCLC (12). Gefitinib, a first-generation EGFR-TKI, is a reversible inhibitor to competes with ATP to bind its binding pocket, which prevents EGFR-induced activation of downstream signaling (13). It has been shown to be dramatically effective in NSCLC patients with EGFR-activating mutations (14). However, most patients eventually develop resistance (15). 
Celastrol a pentacyclic triterpenoid (10-Hydroxy2,4a,6a,9,12b,14a-hexamethyl-11-oxo-1,2,3,4,4a,5,6,6a,11,12b, 13,14,14a,14b-tetradecahydro-picene-2-carboxylic acid), extracted from roots of Tripterygium wilfordii Hook.f. and Celastrus regelii, $\mathrm{L}$. has been reported to have antioxidant and anti-inflammatory activities (16-18). The anticancer effect of celastrol has also been demonstrated by induction of apoptosis and inhibition of cell growth, proliferation and metastasis (19-22).

In this study, we first examined the effect of celastrol on Axl protein level and then if Axl inhibition by celastrol could reverse gefitinib sensitivity of EGFR mutant NSCLC cells with the acquired resistance. We observed the effect of celastrol, gefitinib, or both drugs on Axl protein level, cell proliferation, and migration in gefitinib-resistant cells.

\section{Materials and methods}

Reagents and antibodies. Celastrol was obtained from Sigma-Aldrich (Merck KGaA, Darmstadt, Germany). PC-9 cells were purchased from the American Type Culture Collection (Manassas, VA, USA). Primers for Axl were synthesized by the domestic company, Bioneer Corp. (Daejeon, Korea). TRI reagent was obtained from Solgent Co., Ltd. (Daejeon, Korea). AmpliTaq DNA polymerase and Lipofectamine 2000 were obtained from Roche Diagnostics Corp. (Indianapolis, IN, USA) and Invitrogen (Thermo Fisher Scientific, Inc., Waltham, MA, USA), respectively. For Western blot analysis, specific antibodies against Axl and GAPDH, as well as secondary antibodies were obtained from Santa Cruz Biotechnology, Inc., (Dallas, TX, USA).

Cell culture and establishment of gefitinib-resistant cells. The subline of PC-9 cells which are resistant to gefitinib (PC-9/GR) were established by stepwise exposure of the parental cells to escalating concentrations of gefitinib (ranging from 0.5 to $10 \mu \mathrm{M}$ ). Both PC-9 and PC-9/GR cells were grown in RPMI-1640 (Gibco BRL) containing 10\% FBS, $2 \mathrm{mM}$ L-glutamine, $10 \mathrm{U} / \mathrm{ml}$ penicillin and $10 \mathrm{~g} / \mathrm{ml}$ streptomycin at $37^{\circ} \mathrm{C}$ in $5 \% \mathrm{CO}_{2}$ in a water-saturated atmosphere.

Cell viability measurement. To measure cell viability, the number of viable cells was counted using Trypan blue. Briefly, $3 \times 10^{3}$ cells were seeded into $60-\mathrm{mm}$ culture dish, grown overnight and then treated with the indicated concentrations of gefitinib alone, celastrol alone, or the two drugs in combination for $24 \mathrm{~h}$. After treatment, cells were harvested and stained with $0.4 \%$ Trypan blue solution. Dye-excluding viable cells were counted under the microscope. Cell viability was also expressed as a percentage of the viable cells with respect to untreated control cells.

Colony formation assay. PC-9 or PC-9/GR cells were seeded into 24 well plates $\left(1 \times 10^{2}\right.$ cells/well) and treated with the indicated concentrations of gefitinib alone, celastrol alone, or the two drugs in combination. Cells were then cultured for the next 7 to 10 days to form colonies. Colonies of $>50$ cells were stained with crystal violet (in 60\% methanol; Junsei Chemical Co., Tokyo, Japan) and images were acquired using the RAS-3000 Image Analysis System (FujiFilm, Tokyo, Japan).
Western blot analysis. Total cell lysates from PC-9 and PC-9/GR treated with the indicated concentrations of celastrol alone, gefitinib alone or the two drugs in combination were prepared using lysis buffer [1\% Triton X-100, $50 \mathrm{mM}$ Tris (pH 8.0), $150 \mathrm{mM} \mathrm{NaCl}, 1 \mathrm{mM}$ PMSF, $1 \mathrm{mM} \mathrm{Na} \mathrm{VO}_{4}$, and protease inhibitor cocktail]. Untreated cells were used as controls. Protein concentrations were determined using Bio-Rad protein assays. Proteins from the cell lysates were separated by $10 \%$ SDS-PAGE, and electrotransferred onto nitrocellulose membranes. The membranes were blocked for $30 \mathrm{~min}$ at room temperature in Tris-buffered saline with $0.05 \%$ Tween-20 (TTBS) containing 5\% non-fat dry milk, and then incubated with TTBS containing a primary antibody for $4 \mathrm{~h}$ at room temperature. After $3 \times 10$ min washes in TTBS, the membranes were incubated with peroxidase-conjugated secondary antibody for $1 \mathrm{~h}$. Following 3 additional 10-min washes with TTBS, the protein bands of interest were visualized using an enhanced chemiluminescence detection system (Amersham $^{\mathrm{TM}}$ ECL $^{\mathrm{TM}}$ Prime Western Blotting Detection Reagent; GE Healthcare, Piscataway, NJ, USA).

Reverse transcription-polymerase chain reaction (RT-PCR). Using TRI reagent, total RNAs from PC-9 and PC-9/GR cells were extracted and subjected to cDNA synthesis and PCR. The specific primers were as follows: Axl sense, 5'-AACCTTCAA CTCCTGCCTTCTCG-3' and antisense, 5'-CAGCTTCTCCTT CAGCTCTTCAC-3'; GAPDH sense, 5'-GGAGCCAAAAGG GTCATCAT-3' and antisense, 5'-GTGATGGCATGGACTGTG GT-3'. The mRNA level of Axl was normalized to that of GAPDH.

Flow cytometry. To determine Axl protein level present in plasma membrane, FACS analysis was performed. Cells $\left(1 \times 10^{6}\right)$ were stained with $5 \mathrm{ug} / \mathrm{ml}$ anti-Axl PE-conjugated antibody for $15 \mathrm{~min}$ and subjected to flow cytometry using a Becton-Dickinson FACS Caliber and analyzed by Cell Quest software (Becton-Dickinson, San Jose, CA, USA).

In vitro migration assay. Cells $\left(2-4 \times 10^{4}\right.$ cells/well) were seeded into collagen I-coated 96-well plates (Essen BioScience, Ann Arbor, MI, USA) and grown overnight to form monolayer. According to the manufacturer's instructions, scratch wound was made onto monolayer and the detached cells were removed by washing with PBS. Cells were then treated with the indicated concentrations of gefitinib alone, celastrol alone, or the two drugs in combination for $48 \mathrm{~h}$ and the wound width was measured every $4 \mathrm{~h}$ using IncuCyte software (Essen BioScience, Ann Arbor, MI, USA).

Statistical analysis. Data were expressed as the means \pm SD of triplicate samples or at least three independent experiments. To determine statistical significance, Student's t-test or one-way analysis of variance followed by Dunnett's multiple comparisons test was performed using GraphPad Prism version 8.0.0 (GraphPad Software, San Diego, California USA) with a P-value threshold of $<0.05$.

\section{Results}

Axl expression is upregulated in gefitinib-resistant cells. Majority of patients treated with gefitinib, an epidermal growth 
factor receptor (EGFR) tyrosine kinase inhibitor (TKI), develop resistance $(15,23)$. To investigate the characteristics and underlying mechanisms of the acquired resistance of gefitinib, we first established gefitinib-resistant cells, PC-9/GR, by treating parental PC-9 cells with increasing concentrations of gefitinib in stepwise manner.

PC-9/GR cells were found to have no prominent changes in size and shape compared to the parental PC-9 cells, indicating that in morphological features, PC-9/GR cells are not quite different from parental cells (data not shown). The sensitivities to gefitinib of these cells were assessed by the comparison of the viability of PC-9 and PC-9/GR cells under gefitinib treatment ranging from 0.01 to $1,000 \mu \mathrm{M}$. Cell proliferation of both PC-9 and PC-9/GR cells was decreased in dose-dependent manner, but upon higher than $10 \mathrm{M}$ gefitinib, PC-9 cells were far more sensitive to gefitinib than PC-9/GR cells, indicating that PC-9/GR cells had acquired chemoresistance to gefitinib (Fig. 1A).

Colony formation assay was further confirmed the acquired gefitinib resistance of PC-9/GR cells. Both PC-9 and PC-9/GR cells were treated with the indicated concentrations of gefitinib and then grown for next 7 days. As shown in Fig. 1B, gefitinib treatment decreased the clonogenicity of these cells in a dose-dependent manner. Of note, parental PC-9 was found to form significantly less number of colonies, in contrast to PC-9/GR cells in response to gefitinib treatment. Taken together, these results indicate PC-9/GR cells with gefitinib resistance was established.

Since overexpression and activation of Axl, a receptor tyrosine kinase (RTK), have been reported to be associated with resistance to various TKIs (24-27), we next examined if the acquisition of gefitinib resistance has an effect on the expression of Axl in PC-9/GR cells. Both protein and mRNA levels of Axl in PC-9 and PC-9/GR cells were examined. As shown in Fig. 1C, Western blot analysis revealed that in PC-9/GR cells, Axl protein level was significantly increased compared to parental PC-9 cells. Up-regulation of Axl expression in PC-9/GR cell was further confirmed by RT-PCR. Axl mRNA level in PC-9/GR cells was found to be much higher than that in PC-9 cells, which is consistent with Western blot analysis result.

Additionally, FACS analysis was performed to determine Axl protein level present in plasma membrane, since Axl is a cell surface receptor tyrosine kinase with single transmembrane domain. We found that membrane-bound Axl protein level in PC-9/GR cells was higher than that of parental PC-9 cells (Fig. 1D). All these results indicate that Axl expression is increased in gefitinib-resistant PC-9/GR cells.

Celastrol reduces Axl expression and proliferation in both parental and gefitinib-resistant cells. There have been several molecular targets of celastrol to regulate various cellular responses such as cell cycle, angiogenesis, and inflammation (19), but its effect on Axl expression was not examined yet. We first observed Axl protein level in PC-9 and PC-9/GR cells treated with celastrol. As shown in Fig. 2A, Axl protein levels in these cells treated with celastrol was significantly and dose-dependently reduced, indicating that $\mathrm{Axl}$ is a novel target of celastrol.

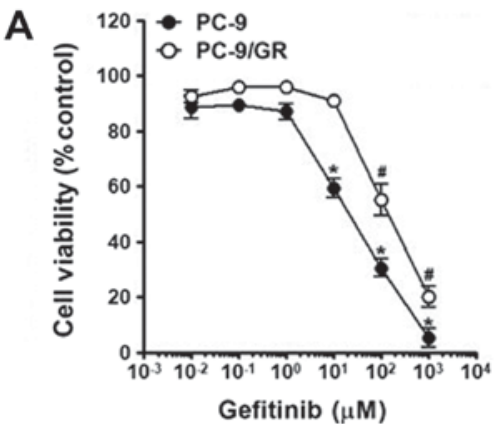

B PC.9

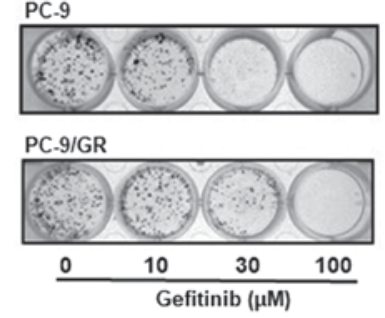

C
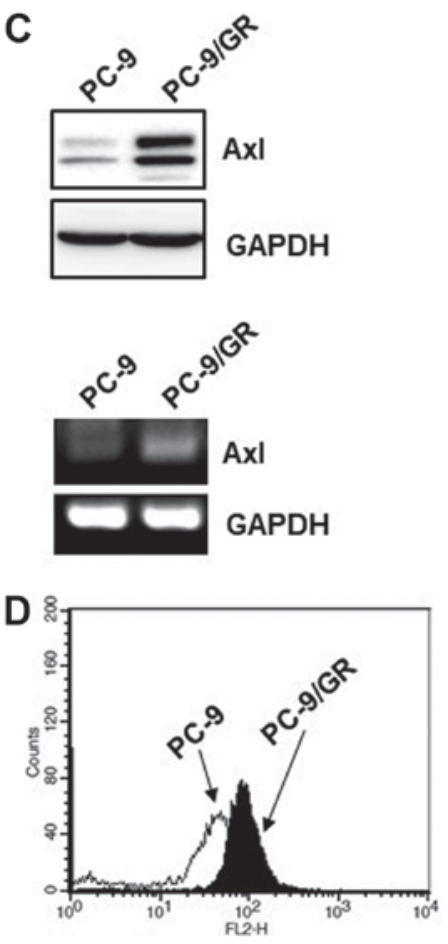

Figure 1. Axl expression is transcriptionally upregulated in gefitinib-resistant EGFR mutant NSCLC cells. PC-9 cells were exposed to increasing concentrations of gefitinib in a stepwise manner to establish gefitinib-resistant variants, PC-9/GR cells. (A) Parental PC-9 cells and gefitinib-resistant PC-9/GR cells were treated with 0.01 to $1,000 \mu \mathrm{M}$ gefitinib for $24 \mathrm{~h}$. The number of viable cells were counted using trypan blue exclusion assay. Each error bar represents the mean \pm standard deviation of at least three replicates. ${ }^{*} \mathrm{P}<0.05$ vs. $10^{-2}$ gefitinib-treated group in $\mathrm{PC}-9$; ${ }^{~} \mathrm{P}<0.05$ vs. $10^{-2}$ gefitinib-treated group in PC-9/GR. (B) Cells $\left(1 \times 10^{3}\right.$ cells/well) were seeded onto 96-well plates, treated with the indicated concentrations of gefitinib and allowed to grow for 7 days until colonies formed. The colonies were visualized by crystal violet staining. The data shown is representative of at least three independent experiments. (C) Axl protein levels in PC-9 and PC-9/GR cells were determined by western blot analysis. GAPDH was used as a loading control. For RT-PCR, total RNAs from PC-9 and PC-9/GR cells were isolated and used to amplify Axl mRNA. As a normalization control, GAPDH mRNA was also amplified using RT-PCR. The data shown is a representative of three independent experiments. (D) To determine the Axl protein level in cell surface, cells $\left(3 \times 10^{4}\right)$ were stained with anti-Axl PE-conjugated antibody and then analyzed using flow cytometry. Results shown are representative of three independent experiments. GR, gefitinib-resistant; PE, phycoerythrin; RT-PCR, reverse transcription-polymerase chain reaction. 
A PC-9

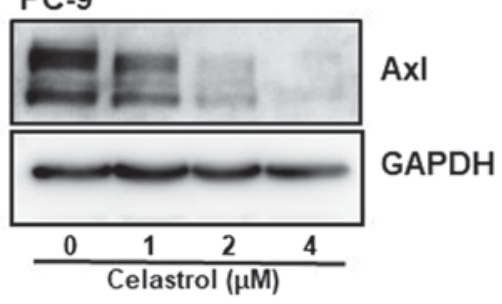

PC-9/GR
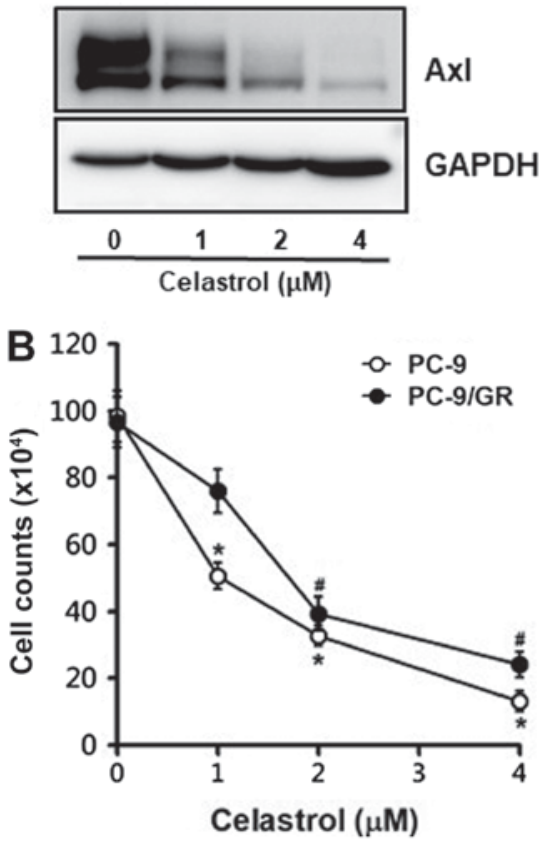

C PC-9

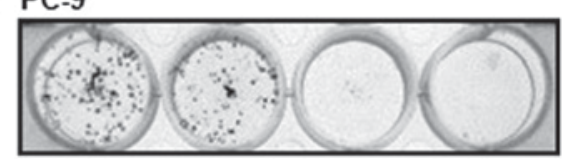

PC-9/GR

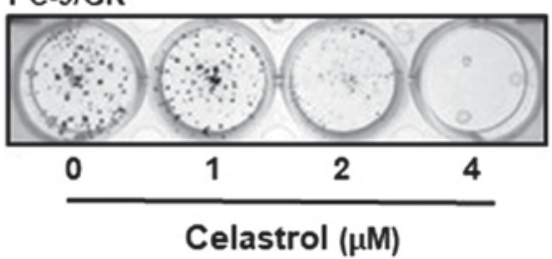

Figure 2. Celastrol reduces Axl protein expression level and inhibits the proliferation of parental and gefitinib-resistant cells. PC-9 and PC-9/GR cells $\left(5 \times 10^{5}\right.$ cells/dish) were seeded onto $100-\mathrm{mm}$ dishes, grown overnight and treated with 1,2 and $4 \mu \mathrm{M}$ celastrol for $24 \mathrm{~h}$ and then cells were harvested. (A) Total cell lysates were prepared and used to determine Axl protein expression levels using western blot analysis. GAPDH was used as a loading control. Result shown is a representative of three independent experiments. (B) To count the number of viable cells, trypan blue exclusion assay was conducted. Data are represented as mean \pm standard deviation of at least three independent experiments. ${ }^{*} \mathrm{P}<0.05$ vs. untreated group in PC-9; ${ }^{\#} \mathrm{P}<0.05$ vs. untreated group in PC-9/GR. (C) Cells $\left(1 \times 10^{3}\right.$ cells/well) were seeded into 24 -well plates and treated with 1, 2 and $4 \mu \mathrm{M}$ celastrol and grown for 7 days. The colonies were visualized by crystal violet staining. The data shown is representative of at least three independent experiments. GR, gefitinib-resistant.

In consistent with the Western blot result shown the inhibitory effect of celastrol on Axl expression, celastrol treatment of these cells was found to result in the dose-dependent reduction of cell viability (Fig. 2B). Of note, survival rate of PC-9 and PC-9/GR cells treated with $4 \mu \mathrm{M}$ celastrol were found to be only 13 and $24 \%$, respectively.

The anti-proliferative effect of celastrol was further confirmed by colony formation assay. Treatment of cells with celastrol suppressed the clonogenicity in dose-dependent manner (Fig. 2C). Interestingly, PC-9/GR cells exposed to $4 \mu \mathrm{M}$ celastrol formed more colonies compared to parental PC-9 cells, which seemed to be resulted from the elevated Axl level of PC-9/GR cells.

Celastrol and gefitinib cooperatively diminish Axl protein level, cell proliferation, and cell migration. Since Axl protein level is pretty elevated in PC-9/GR cells, we investigated if Axl inhibition by celastrol could reverse gefitinib sensitivity of PC-9/GR cells. To do this end, we observed the effect of celastrol and gefitinib in combination on Axl protein level, cell viability and cell migration.

As shown in Fig. 3A, cotreatment of PC-9/GR cells with celastrol and gefitinib synergistically decreased Axl protein level compared to treatment of the cells with celastrol or gefitinib alone, indicating that celastrol augments gefitinib activity to reduce Axl expression. Cell viability was also found to be additionally attenuated by treatment of cells with celastrol and gefitinib in combination compared to treatments of cells with celastrol or gefitinib alone (Fig. 3B). This combinatorial effect of celastrol and gefitinib to inhibit cell proliferation was further confirmed by colony formation assay. As shown in Fig. 3C, PC-9/GR cells exposed to celastrol and gefitinib formed less colonies than cells treated with single drug. Taken together, these results indicate that celastrol might sensitize cytotoxicity of gefitinib to overcome gefitinib resistance of PC-9/GR cells via Axl targeting.

We then examined the effect of combination of celastrol and gefitinib on cell migration via wound healing assays. Compared to treatments of PC-9/GR cells with celastrol or gefitinib alone, the combined treatment of cells with celastrol and gefitinib was found to result in much less reduction in wound surface area over time (Fig. 4A). Of note, the percentage reduction in wound area was $67.6 \%$ (celastrol alone), $64.6 \%$ (gefitinib alone), and $42.3 \%$ (cotreatment of two drugs), respectively (Fig. 4B). Taken together, these results indicate that celastrol seems to increase the susceptibility of PC-9/GR cells to gefitinib via Axl targeting.

\section{Discussion}

Non-small cell lung cancer (NSCLC) patients with activating mutation in the epidermal growth factor receptor (EGFR) gene are a much more sensitive to EGFR tyrosine kinase inhibitors (TKIs) compared to patients with wild-type EGFR (6). The first-generation EGFR-TKIs, gefitinib and erlotinib, has been demonstrated to be highly effective and can even be prescribed as first-line therapy of NSCLC harboring activating EGFR mutation. However, the resistance to these TKIs ultimately emerged in majority of patients. Thus, identification of secondary mutations and understanding of the molecular mechanisms associated with the acquired resistance are urgent and essential to overcome relapsed NSCLCs.

In this study, PC-9 cells with targetable driver mutation that is activating in-frame deletion in exon 19 of EGFR 
A

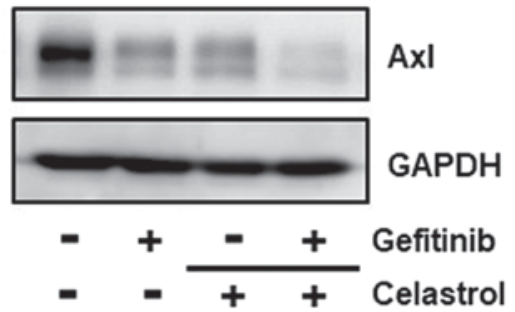

B
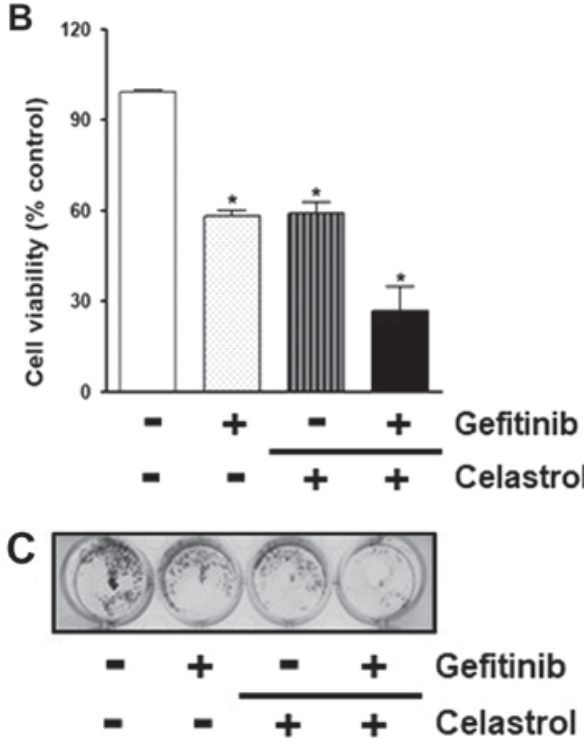

Figure 3. Celastrol and gefitinib cooperatively diminish Axl protein expression level and cell proliferation. PC-9/GR cells $\left(5 \times 10^{5}\right.$ cells/dish) were seeded onto $100-\mathrm{mm}$ dishes, grown overnight and treated with $2 \mu \mathrm{M}$ celastrol, $10 \mu \mathrm{M}$ gefitinib or combination of celastrol and gefitinib for $24 \mathrm{~h}$. (A) For western blot analysis, the total cell lysates were prepared to determine Axl protein expression level. GAPDH was used as a loading control and results shown are representative of at least three independent experiments. (B) To assess cell viability, trypan blue exclusion assay was conducted. Data are represented as mean \pm standard of at least three independent experiments. ${ }^{*} \mathrm{P}<0.05$ vs. untreated group. (C) For colony formation assay, cells $\left(1 \times 10^{3}\right.$ cells/well) were seeded in 24 -well plates and treated with the indicated each drug or combination of celastrol and gefitinib and allowed to grow for 7 days. Colonies were visualized by crystal violet staining. The data shown is representative of three independent experiments. GR, gefitinib-resistant.

were exposed to increasing concentrations of gefitinib to establish gefitinib-resistant, PC-9/GR, cells. As shown in Fig. 1C and D, Axl protein and mRNA levels in PC-9/GR cells were found to be significantly increased, indicating that long-term exposure of PC-9 cells resulted in outgrowth of gefitinib-resistant cells by up-regulation of Axl expression. Axl is a member of Tyro/Axl/Mer (TAM) family of receptor tyrosine kinases (RTKs) (28). Activation of Axl has been known to be required for cell growth, migration, proliferation, and inhibition of apoptosis in many cancers (26,29-31). In consistant with our results, the induction of Axl expression has already been demonstrated in gefitinib- or erlotinib-resistant EGFR-mutant NSCLC cells or NSCLC tumor xenografts, respectively $(25,32,33)$. In addition, a number of studies reported that the acquisition of resistance to other TKIs, including imatinib and nilotinib is correlated with abnormal activation and/or overexpression of TAM RTKs in various cancers such as chronic myeloid leukemia cells $(26,34)$. Therefore, the inhibition of Axl or other members of TAM RTKs which were aberrantly regulated in TKI-resistant cells
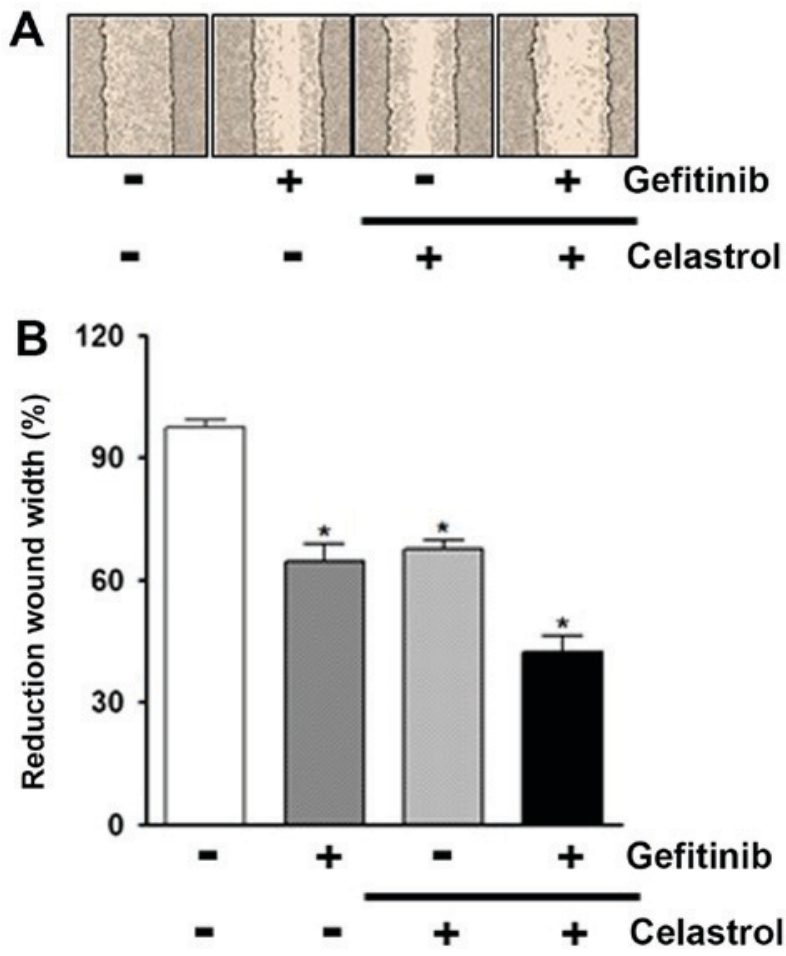

Figure 4. Celastrol and gefitinib synergistically suppresses cell migration. PC-9/GR $\left(5 \times 10^{4}\right)$ cells were seeded into 96 well plates and grown overnight. Scratch wound was made onto monolayer of cells. (A) Cells were treated with $2 \mu \mathrm{M}$ celastrol, $10 \mu \mathrm{M}$ gefitinib or combination of celastrol and gefitinib. The initial wound is marked in dark gray. Result shown is a representative of three independent experiments. (B) The percentages of wound width reduction were calculated at $24 \mathrm{~h}$ post-incubation using IncuCyte software. Data are expressed as the mean \pm standard deviation of triplicate samples. ${ }^{*} \mathrm{P}<0.05$ vs. the control group. GR, gefitinib-resistant.

might recover susceptibility to TKIs, which subsequently overcome the acquired resistance.

Celastrol was found to inhibit proliferation of both parental cells, PC-9, and gefitinib-resistant cells, PC-9/GR (Fig. 2A and B). In consistent with our data, the anticancer effect of celastrol was observed in many cancers, including breast, cervical, colon, gastric, prostate cancers (35-39). Furthermore, chemosensitizing effect of celastrol have also been revealed in cisplatin- and gefitinib-resistant cells (19,40-42), since PC-9/GR cells treated with celastrol and gefitinib in combination synergistically decreased Axl protein level, cell proliferation, and cell migration (Figs. 3 and 4). The cooperative efficacy of combined therapy with celastrol and several anticancer agents has been reported in doxorubicin-resistant colon cancer cells, cisplatin/gefitinib-resistant NSCLC cells via induction of apoptosis, inhibition of JNK/ATF2 pathway, and autophagic degradation of EGFR, respectively $(40,42)$. Interestingly, Wang et al (41) reported that the combined treatment with celastrol and EGFR-TKIs such as gefitinib and erlotinib was found to be more effective to inhibit invasion of NSCLC cells with an EGFR T790M mutation, while EGFR-TKIs alone had little effect on it. Increasing evidence indicates that celastrol might be a potent candidate to augment the sensitivity of conventional chemotherapeutic agents in numerous cancers.

In summary, our data indicate that celastrol downregulates Axl expression in parental and gefitinib-resistant NSCLC cells. 
Thus, Axl seems to be a novel target of celastrol to inhibit cell proliferation and to increase the susceptibility of gefitnib-resistance cells to gefitinib, which lead to overcome chemoresistance.

\section{Acknowledgements}

Not applicable.

\section{Funding}

The present study was supported by the 2015 Yeungnam University Research Grant (grant no. 215A480013).

\section{Availability of data and materials}

The datasets used and/or analyzed during the current study are available from the corresponding author on reasonable request.

\section{Authors' contributions}

YJL, SYK and CL were equally responsible for the establishment of the conception and design of study, and the acquisition, analysis and interpretation of data. YJL, SYK and CL read and approved the final manuscript.

\section{Ethics approval and consent to participate}

Not applicable.

\section{Patient consent for publication}

Not applicable.

\section{Competing interests}

The authors declare that they have no competing interests.

\section{References}

1. Siegel RL, Miller KD and Jemal A: Cancer statistics, 2018. CA Cancer J Clin 68: 7-30, 2018.

2. Ettinger DS, Akerley W, Borghaei H, Chang AC, Cheney RT, Chirieac LR, D'Amico TA, Demmy TL, Govindan R, Grannis FW Jr, et al: Non-small cell lung cancer, version 2.2013. J Natl Compr Canc Netw 11: 645-653; quiz 653, 2013.

3. Hamilton G and Rath B: Pharmacogenetics of platinum-based chemotherapy in non-small cell lung cancer: Predictive validity of polymorphisms of ERCC1. Expert Opin Drug Metab Toxicol 14: 17-24, 2018.

4. Forde PM and Ettinger DS: Targeted therapy for non-small-cell lung cancer: Past, present and future. Expert Rev Anticancer Ther 13: 745-758, 2013.

5. Cabanero M, Sangha R, Sheffield BS, Sukhai M, Pakkal M, Kamel-Reid S, Karsan A, Ionescu D, Juergens RA, Butts C and Tsao MS: Management of EGFR-mutated non-small-cell lung cancer: Practical implications from a clinical and pathology perspective. Curr Oncol 24: 111-119, 2017.

6. González-Larriba JL, Lázaro-Quintela M, Cobo M, Domine M, Majem $\mathrm{M}$ and García-Campelo R: Clinical management of epidermal growth factor receptor mutation-positive non-small cell lung cancer patients after progression on previous epidermal growth factor receptor tyrosine kinase inhibitors: The necessity of repeated molecular analysis. Transl Lung Cancer Res 6 (Suppl 1): S21-S34, 2017.

7. Wada T, Qian XL and Greene MI: Intermolecular association of the p185neu protein and EGF receptor modulates EGF receptor function. Cell 61: 1339-1347, 1990.
8. Carraway KL III and Cantley LC: A neu acquaintance for erbB3 and erbB4: A role for receptor heterodimerization in growth signaling. Cell 78: 5-8, 1994.

9. Yarden Y: The EGFR family and its ligands in human cancer. Signaling mechanisms and therapeutic opportunities. Eur J Cancer 37 (Suppl 4): S3-S8, 2001.

10. Zhang H, Berezov A, Wang Q, Zhang G, Drebin J, Murali R and Greene MI: ErbB receptors: From oncogenes to targeted cancer therapies. J Clin Invest 117: 2051-2058, 2007.

11. Seshacharyulu P, Ponnusamy MP, Haridas D, Jain M, Ganti AK and Batra SK: Targeting the EGFR signaling pathway in cancer therapy. Expert Opin Ther Targets 16: 15-31, 2012.

12. Arteaga C: Targeting HER1/EGFR: A molecular approach to cancer therapy. Semin Oncol 30 (3 Suppl 7): S3-S14, 2003.

13. Pao W, Miller V, Zakowski M, Doherty J, Politi K, Sarkaria I, Singh B, Heelan R, Rusch V, Fulton L, et al: EGF receptor gene mutations are common in lung cancers from 'never smokers' and are associated with sensitivity of tumors to gefitinib and erlotinib. Proc Natl Acad Sci USA 101: 13306-13311, 2004.

14. Araki T, Yashima H, Shimizu K, Aomori T, Hashita T, Kaira K, Nakamura $T$ and Yamamoto K: Review of the treatment of non-small cell lung cancer with gefitinib. Clin Med Insights Oncol 6: 407-421, 2012.

15. Xu Y, Liu H, Chen J and Zhou Q: Acquired resistance of lung adenocarcinoma to EGFR-tyrosine kinase inhibitors gefitinib and erlotinib. Cancer Biol Ther 9: 572-582, 2010.

16. Guan Y, Cui ZJ, Sun B, Han LP, Li CJ and Chen LM: Celastrol attenuates oxidative stress in the skeletal muscle of diabetic rats by regulating the AMPK-PGC1 $\alpha$-SIRT3 signaling pathway. Int J Mol Med 37: 1229-1238, 2016.

17. Ju SM, Youn GS, Cho YS, Choi SY and Park J: Celastrol ameliorates cytokine toxicity and pro-inflammatory immune responses by suppressing NF- $\mathrm{KB}$ activation in RINm5F beta cells. BMB Rep 48: 172-177, 2015.

18. Li X, Wu N, Zou L and Jia D: Protective effect of celastrol on myocardial ischemia-reperfusion injury. Anatol J Cardiol 18: 384-390, 2017.

19. Kashyap D, Sharma A, Tuli HS, Sak K, Mukherjee T and Bishayee A: Molecular targets of celastrol in cancer: Recent trends and advancements. Crit Rev Oncol Hematol 128: 70-81, 2018.

20. Mi C, Shi H, Ma J, Han LZ, Lee JJ and Jin X: Celastrol induces the apoptosis of breast cancer cells and inhibits their invasion via downregulation of MMP-9. Oncol Rep 32: 2527-2532, 2014.

21. Rajendran P, Li F, Shanmugam MK, Kannaiyan R, Goh JN, Wong KF, Wang W, Khin E, Tergaonkar V, Kumar AP, et al: Celastrol suppresses growth and induces apoptosis of human hepatocellular carcinoma through the modulation of STAT3/JAK2 signaling cascade in vitro and in vivo. Cancer Prev Res (Phila) 5: 631-643, 2012.

22. Abbas S, Bhoumik A, Dahl R, Vasile S, Krajewski S, Cosford ND and Ronai ZA: Preclinical studies of celastrol and acetyl isogambogic acid in melanoma. Clin Cancer Res 13: 6769-6778, 2007.

23. Yano S, Yamaguchi M and Dong RP: EGFR tyrosine kinase inhibitor 'gefitinib (Iressa)' for cancer therapy. Nihon Yakurigaku Zasshi 122: 491-497, 2003 (In Japanese).

24. Wu F, Li J, Jang C, Wang J and Xiong J: The role of Axl in drug resistance and epithelial-to-mesenchymal transition of non-small cell lung carcinoma. Int J Clin Exp Pathol 7: 6653-6661, 2014.

25. Bae SY, Hong JY, Lee HJ, Park HJ and Lee SK: Targeting the degradation of AXL receptor tyrosine kinase to overcome resistance in gefitinib-resistant non-small cell lung cancer. Oncotarget 6: 10146-10160, 2015.

26. Dufies M, Jacquel A, Belhacene N, Robert G, Cluzeau T, Luciano F, Cassuto JP, Raynaud S and Auberger P: Mechanisms of AXL overexpression and function in imatinib-resistant chronic myeloid leukemia cells. Oncotarget 2: 874-885, 2011.

27. Rho JK, Choi YJ, Kim SY, Kim TW, Choi EK, Yoon SJ, Park BM, Park E, Bae JH, Choi CM and Lee JC: MET and AXL inhibitor NPS-1034 exerts efficacy against lung cancer cells resistant to EGFR kinase inhibitors because of MET or AXL activation. Cancer Res 74: 253-262, 2014.

28. Lemke G: Biology of the TAM receptors. Cold Spring Harb Perspect Biol 5: a009076, 2013.

29. Martinelli E, Martini G, Cardone C, Troiani T, Liguori G, Vitagliano D, Napolitano S, Morgillo F, Rinaldi B, Melillo RM, et al: AXL is an oncotarget in human colorectal cancer. Oncotarget 6: 23281-23296, 2015.

30. Qu X, Liu J,Zhong X, Li X and Zhang Q: Role of AXL expression in non-small cell lung cancer. Oncol Lett 12: 5085-5091, 2016. 
31. Rankin EB, Fuh KC, Taylor TE, Krieg AJ, Musser M, Yuan J, Wei K, Kuo CJ, Longacre TA and Giaccia AJ: AXL is an essential factor and therapeutic target for metastatic ovarian cancer. Cancer Res 70: 7570-7579, 2010.

32. Giles KM, Kalinowski FC, Candy PA, Epis MR, Zhang PM Redfern AD, Stuart LM, Goodall GJ and Leedman PJ: Axl mediates acquired resistance of head and neck cancer cells to the epidermal growth factor receptor inhibitor erlotinib. Mol Cancer Ther 12: 2541-2558, 2013.

33. Wang Y,Xia H,Zhuang Z, Miao L, Chen X and Cai H: Axl-altered microRNAs regulate tumorigenicity and gefitinib resistance in lung cancer. Cell Death Dis 5: e1227, 2014.

34. Gioia R, Tregoat C, Dumas PY, Lagarde V, Prouzet-Mauléon V, Desplat V, Sirvent A, Praloran V, Lippert E, Villacreces A, et al: CBL controls a tyrosine kinase network involving AXL, SYK and LYN in nilotinib-resistant chronic myeloid leukaemia. J Pathol 237: 14-24, 2015.

35. Tang WJ, Wang J, Tong X, Shi JB, Liu XH and Li J: Design and synthesis of celastrol derivatives as anticancer agents. Eur J Med Chem 95: 166-173, 2015.

36. Kuchta K, Xiang Y, Huang S, Tang Y, Peng X, Wang X, Zhu Y, $\mathrm{Li} \mathrm{J}, \mathrm{Xu}$ J, Lin Z and Pan T: Celastrol, an active constituent of the TCM plant Tripterygium wilfordii Hook.f., inhibits prostate cancer bone metastasis. Prostate Cancer Prostatic Dis 20: 156-164, 2017.
37. Bufu T, Di X, Yilin Z, Gege L, Xi C and Ling W: Celastrol inhibits colorectal cancer cell proliferation and migration through suppression of MMP3 and MMP7 by the PI3K/AKT signaling pathway. Anticancer Drugs 29: 530-538, 2018.

38. Lee HW, Jang KS, Choi HJ, Jo A, Cheong JH and Chun KH: Celastrol inhibits gastric cancer growth by induction of apoptosis and autophagy. BMB Rep 47: 697-702, 2014.

39. Zhou Y,Li W, Wang M, Zhang H, Tong X and Xiao Y: Competitive profiling of celastrol targets in human cervical cancer HeLa cells via quantitative chemical proteomics. Mol Biosyst 13: 83-91, 2016.

40. Xu SW, Law BY, Mok SW, Leung EL, Fan XX, Coghi PS, Zeng W, Leung CH, Ma DL, Liu L and Wong VK: Autophagic degradation of epidermal growth factor receptor in gefitinib-resistant lung cancer by celastrol. Int J Oncol 49: 1576-1588, 2016.

41. Wang Y, Liu Q, Chen H, You J, Peng B, Cao F, Zhang X, Chen Q, Uzan G, Xu L and Zhang D: Celastrol improves the therapeutic efficacy of EGFR-TKIs for non-small-cell lung cancer by overcoming EGFR T790M drug resistance. Anticancer Drugs 29: 748-755, 2018.

42. Lo Iacono M, Monica V, Vavalà T, Gisabella M, Saviozzi S, Bracco E, Novello S, Papotti $M$ and Scagliotti GV: ATF2 contributes to cisplatin resistance in non-small cell lung cancer and celastrol induces cisplatin resensitization through inhibition of JNK/ATF2 pathway. Int J Cancer 136: 2598-2609, 2015. 